\title{
The Role of Magnetic Resonance Imaging in Diagnosis and Prognosis of Rheumatoid Arthritis
}

\author{
MOHSEN G.H. ISMAIL, M.D.; AHMED EL SHIMY, M.D. and ASMAA ZAID BAHLOL SAMIN, M.Sc. \\ The Department of Radiodiagnosis, Faculty of Medicine, A in Shams University
}

\begin{abstract}
Background: Rheumatoid arthritis (RA) is one of the most common forms of arthritis. A progressive joint damage occurs early in the disease course even before being detected in conventional imaging, and eventually leads to irreversible joint deformity and severe functional impairment. Routinely, clinical assessment and laboratory investigations as well as conventional radiology are used for diagnosis of RA, however; they are not fair enough to establish early diagnosis and can't provide information about the disease activity. Estimating the disease activity at onset is fundamental to identify the prognosis of RA.
\end{abstract}

Aim of Study: The aim of this study was to demonstrate the importance of MRI in detecting early signs of arthritis helping in early diagnosis of rheumatoid arthritis, and its ability to assess the degree of inflammatory activity as a prognostic parameter for the development of subsequent bone destruction.

Patients and Methods: The twenty-five patients included in this study (14 females, 56\%, and 11 males, 44\%), were diagnosed with rheumatoid arthritis according to the 2010 ACR/EULA classification criteria. The clinically dominant wrist and hand joints were examined by MRI study for detection of pathological signs of RA that include synovitis, BME, and erosions. Twenty (20) patients $(80 \%)$ were found to have synovial thickening. Of which, 12 wrist joints $(60 \%)$ were active, among them 5 joints (25\%) had mild activity, 4 (20\%) had moderate activity and 3 wrist joints had severe activity $(15 \%) .17$ patients had bone marrow edema $(68 \%)$, more frequently at the radius and the lunate. Erosions was seen in 8 joints (32\%). The total positive MRI inflammatory findings, as well as each one of them alone, were compared and correlated with the 2010 ACR/EULA scoring system.

Results: We found significant correlation and agreement between the total MRI positive findings and the 2010 ACR/EULAR criteria used for diagnosing RA, and MRI was more sensitive in detecting synovitis and BME in patients who didn't meet the criteria.

Conclusion: The high sensitivity of MRI in detecting inflammatory signs, and the significant correlation with clinical

Correspondence to: Dr. Mohsen G.H. Ismail, The Department of Radiodiagnosis, Faculty of Medicine, Ain Shams University and laboratory findings, indicates the potential importance of MRI study in diagnosing RA early in its course, and the assessment of disease activity that helps in prediction of joint destruction.

Key Words: Magnetic resonance imaging - Rheumatoid arthritis.

\section{Introduction}

RHEUMATOID arthritis (RA) is one of the most common forms of arthritis. It is a chronic, autoimmune inflammatory joint disease, which primarily involves the small joints of the hands and feet, and affects $0.5-1.0 \%$ of the adult population. Erosive joint damage occurs early in the disease course and patients typically experience joint pain, swelling, tenderness, and stiffness, and they are at risk of developing progressive joint damage, which eventually leads to irreversible joint deformity and severe functional impairment [1].

Without adequate treatment, at 20 years after diagnosis, more than $60 \%$ of patients with RA may develop significant functional impairment, including the need of mobility aids, loss of ability for self-caring, and requirement of joint replacement, or experience loss of independence and require daily care [2]. RA initially involves the synovium of the joints leading to synovial thickening, synovial hyperaemia, and pannus formation; later on, it will progress to bone erosions and destruction and deformity of the joints [3].

According to the American Society for Surgery of the Hand (ASSH, 2017), RA affects the synovial tissue that line and normally lubricate the joints. The synovium becomes inflamed and swollen and erodes the cartilage and bone. The swollen tissue may also stretch the surrounding ligaments, resulting in deformity and instability. The inflammation 
may also spread to the tendons, result in fraying and eventual breaking of the tendons [4]

It is most likely to erode wrist, metacarpophalangeal, and interphalangeal joints. When synovial pannus is formed it may cause gradual erosion of the articular cartilage and bone cortex. So, it is possible to suppress the inflammatory reaction if the disease is recognized early and aggressive treatment is introduced through the first 3 months, known as a therapeutic window. Otherwise, the disease could take on a more chronic, aggressive form, with joint destruction [5]. Over the last two decades, significant improvement has been achieved in its prognosis owing to new strategies for disease management, the emergence of new biologic therapies and better utilization of the conventional disease-modifying antirheumatic drugs. This raises the demand for a diagnosing method that can identify the early signs of the disease [6].

Conventional radiography used as a standard tool for evaluation of RA patients in daily practice. However, the X-ray only shows late signs of disease activity when cartilage or bone is already destructed, while other medical imaging techniques such as ultrasonography (US) and Magnetic resonance imaging (MRI) have been used in RA in order to assess the earlier signs [5]. MRI is increasingly being used in the assessment of rheumatoid arthritis due to its ability to directly visualize the bone and soft tissues in three dimensions and has the potential to measure inflammatory activity and joint destruction. It can detect synovitis, tenosynovitis, bone erosion, and joint effusion [5]. MRI also allows the detection of bone marrow oedema, which is thought to be a precursor for the development of erosions in early RA as well as a marker of active inflammation and can be seen neither on radiographs, ultrasound nor computed tomography (CT). In addition, the multi-plane, multi-slice capability of MRI allows visualization of the area of interest in three orthogonal planes. Therefore, MRI has the advantage of providing details concerning both the bone and surrounding tissues of the joint, which is not shared by any other imaging modality, whilst avoiding ionizing radiation [6]

\section{Aim of the work:}

The aim of our study is to evaluate the utility of magnetic resonance imaging as a sensitive technique which may effectively assist in early detection of rheumatoid arthritis, and identify patients at risk for progressive disease.

\section{Patients and Methods}

This prospective study was conducted on patients referred from the Rheumatology and Rehabilitation outpatient clinic to the MRI department of Ain Shams University, in the period between January 2017-August 2019.

\section{Patients:}

Twenty-five patients were conducted in the study having undifferentiated arthritis and suspected to have early-stage RA. The study included 14 females and 11 males, ages ranged from 20 to 66 years with mean age of 38.64 yrs.

\section{Inclusion criteria:}

- Adult patients with polyarthralgia, suspected to have early stage RA depending on clinical and laboratory findings.

- No apparent deformities of the wrist and hand joints clinically.

- Normal X-ray study.

Exclusion criteria:

- Presence of advanced deformities of the wrist and hand joints detected clinically.

- Contraindications to MRI (e.g.: claustrophobia, pacemakers).

\section{Method:}

$$
\text { to: }
$$$$
\text { All patients included in this study were subjected }
$$

1- Full history taking.

2- Clinical examination by the referring rheumatologist.

3- Laboratory investigations: Patients were subjected to the following laboratory investigations:

- Complete blood picture.

- Serum Rheumatoid Factor.

- Erythrocyte Sedimentation Rate (ESR).

- C-reactive protein (CRP) quantitatively.

4- Plain radiography.

5- MRI study.

MRI study:

Magnetic resonance examination of the second through fifth MCP and wrist joints was performed on the affected side or on the dominant side in the case of symmetric symptoms, was performed using Philips Intera 1.5-T (Philips Healthcare, Best, the Netherlands) with the use of Philips wrist surface coil. Patients were placed in prone position with the hand above the head toward the midline supported by sand bags to maintain its position. 


\section{Evaluation of MRI images:}

$M R$ images are examined and interpreted for presence of the following:

1- Synovitis.

2- Bone Marrow Edema.

3- Bone Erosions.

\section{Statistical analysis:}

Data were collected, revised, coded and entered to the Statistical Package for Social Science (IBM SPSS) version 23 . The quantitative data were presented as Mean, standard deviations (SD) and ranges while qualitative data were presented as number and percentages. So, the $p$-value was considered significant as the following: $p>0.05$ : Non significant, $p<0.05$ : Significant, $p<0.01$ : Highly significant.

\section{Results}

Patients are diagnosed with RA if they score 6 or more points on the 2010 ACR/EULAR classification criteria. Twenty patients had a score $\geq 6$; considered to have $\mathrm{RH}(80 \%)$, and five patients $<6$ did not (20\%). Sixteen patients $(64 \%)$ were seropositive (RF) and nine patients (36\%) were seronegative (RF). Twenty patients were positive for CRP (80\%).
A statistical correlation was done between MRI findings and the ACR/EULAR classification scoring system used for clinical diagnosis of patients with undifferentiated arthritis. There was high grade of significance between the total positive MRI findings and the clinical diagnostic scoring.

A twenty-one years old male has bilateral wrist joint pain and swelling for 8 months, with seropositive RH, CRP and ESR. He was diagnosed to have RA according to ACR/EULAR classification criteria, score 8 .

A twenty-four years old male with pain mainly at wrist joint for 5 months, with seropositive RH, CRP and ESR. He was diagnosed to have RA according to ACR/EULAR classification criteria, score 6.

Table (1): Demographic data.

\begin{tabular}{ll}
\hline Demographic data & No. $=25$ \\
\hline Sex: & \\
Female & $14(56.0 \%)$ \\
Male & $11(44.0 \%)$ \\
Age (years): & \\
Mean \pm SD & $38.64 \pm 14.21$ \\
Range & $20-66$ \\
Symptoms duration (months): & \\
Mean \pm SD & $8.24 \pm 2.50$ \\
Range & $4-13$ \\
\hline
\end{tabular}

Table (2): Relation between results by ACR and findings by MRI .

\begin{tabular}{|c|c|c|c|c|c|c|c|c|c|}
\hline & \multicolumn{4}{|c|}{$\begin{array}{c}\text { Negative ACR/EULAR } \\
\text { Clinical Score }\end{array}$} & \multicolumn{2}{|c|}{$\begin{array}{l}\text { Positive ACR/EULAR } \\
\text { Clinical Score } \\
\end{array}$} & \multirow{2}{*}{$\begin{array}{c}\text { Test } \\
\text { value* }\end{array}$} & \multirow{2}{*}{$\begin{array}{c}p- \\
\text { value }\end{array}$} & \multirow{2}{*}{ Sig. } \\
\hline & & No. & $\%$ & & No. & $\%$ & & & \\
\hline \multicolumn{10}{|l|}{ Synovitis scoring: } \\
\hline Negative & & 3 & 60.0 & & 3 & 15.0 & \multirow[t]{2}{*}{1.563} & \multirow[t]{2}{*}{0.181} & \multirow[t]{2}{*}{ NS } \\
\hline Positive & & 2 & 40.0 & & 17 & 85.0 & & & \\
\hline \multicolumn{10}{|l|}{$B M E:$} \\
\hline Negative & & 3 & 60.0 & & 5 & 25.0 & \multirow[t]{2}{*}{2.252} & \multirow[t]{2}{*}{0.133} & \multirow[t]{2}{*}{ NS } \\
\hline Positive & & 2 & 40.0 & & 15 & 75.0 & & & \\
\hline \multicolumn{10}{|l|}{ Bone Erosion: } \\
\hline Negative & & 5 & 100. & & 12 & 60.0 & \multirow[t]{2}{*}{2.941} & \multirow[t]{2}{*}{0.086} & \multirow[t]{2}{*}{ NS } \\
\hline Positive & & 0 & 0.0 & & 8 & 40.0 & & & \\
\hline \multicolumn{10}{|l|}{ +ve MRI Findings: } \\
\hline Negative & & 3 & 60.0 & & 0 & 0.0 & \multirow[t]{2}{*}{13.636} & \multirow[t]{2}{*}{0.000} & \multirow[t]{2}{*}{ HS } \\
\hline Positive & & 2 & 40.0 & & 20 & 100.0 & & & \\
\hline \multicolumn{10}{|c|}{$\begin{array}{l}p \text {-value }>0.05: \text { Non significant (NS). } \\
p \text {-value }<0.05: \text { Significant }(\mathrm{S}) .\end{array}$} \\
\hline Parameter & TP & $\mathrm{TN}$ & FP & $\mathrm{FN}$ & Accuracy & Sensitivity & Specificity & PPV & NPV \\
\hline Synovitis scoring & 17 & 3 & 2 & 3 & $76.0 \%$ & 80.0 & 45.0 & 85.0 & 40.0 \\
\hline $\mathrm{BME}$ & 15 & 3 & 2 & 5 & $72.0 \%$ & 75.0 & 60.0 & 88.2 & 37.5 \\
\hline Bone Erosion & 8 & 5 & 0 & 12 & $52.0 \%$ & 40.0 & 100.0 & 100.0 & 29.4 \\
\hline +ve MRI Findings & 20 & 3 & 2 & 0 & $92 . \%$ & 100.0 & 60.0 & 90.9 & 100.0 \\
\hline
\end{tabular}



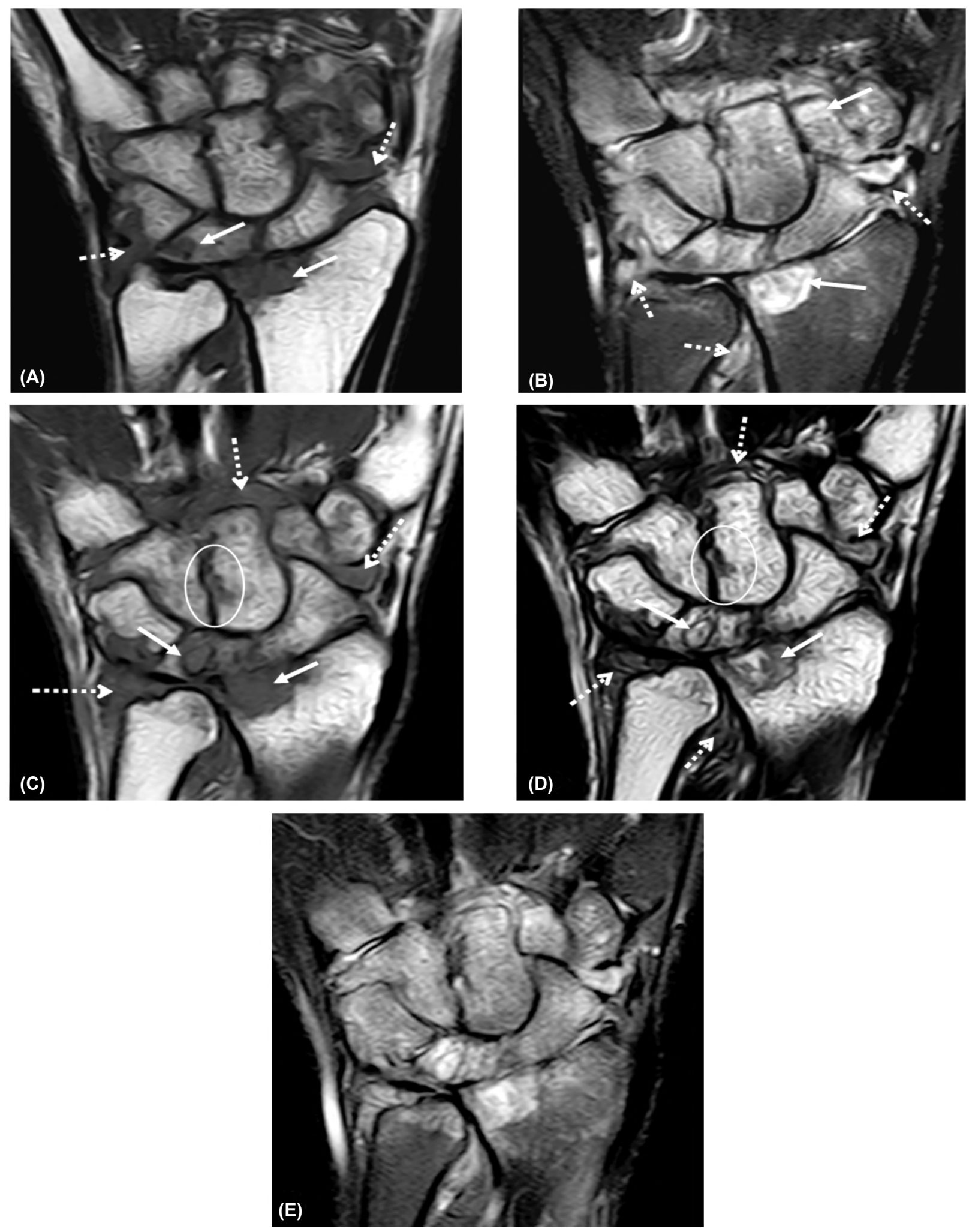

Fig. (1): (A) Coronal T1W image shows bone marrow edema of carpal bones as decreased signal intensity, foci of low signal bone erosions in trapezoid and distal radius. These lesions are better demonstrated after fat suppression by STIR at same section (B). Hyper intensity of carpal bones representing bone marrow edema, and large erosion of medial aspect of radial end. (C) Coronal T1 WI showing thickening of synovium of intermediate signal around carpal bones and radioulnar joint (dotted arrow), with large bone erosion seen in lunate and radial end (arrow). (D) Coronal T2WI showing the thickened synovium as iso-tohyper intensity, and the erosions at lunate and radius has hyperintense signal. (E) Coronal STIR obviously showing bone marrow edema, bone erosions and synovitis. STIR and T2 helped in distinguishing the real bone erosions from normal bone variations (ellipse). 

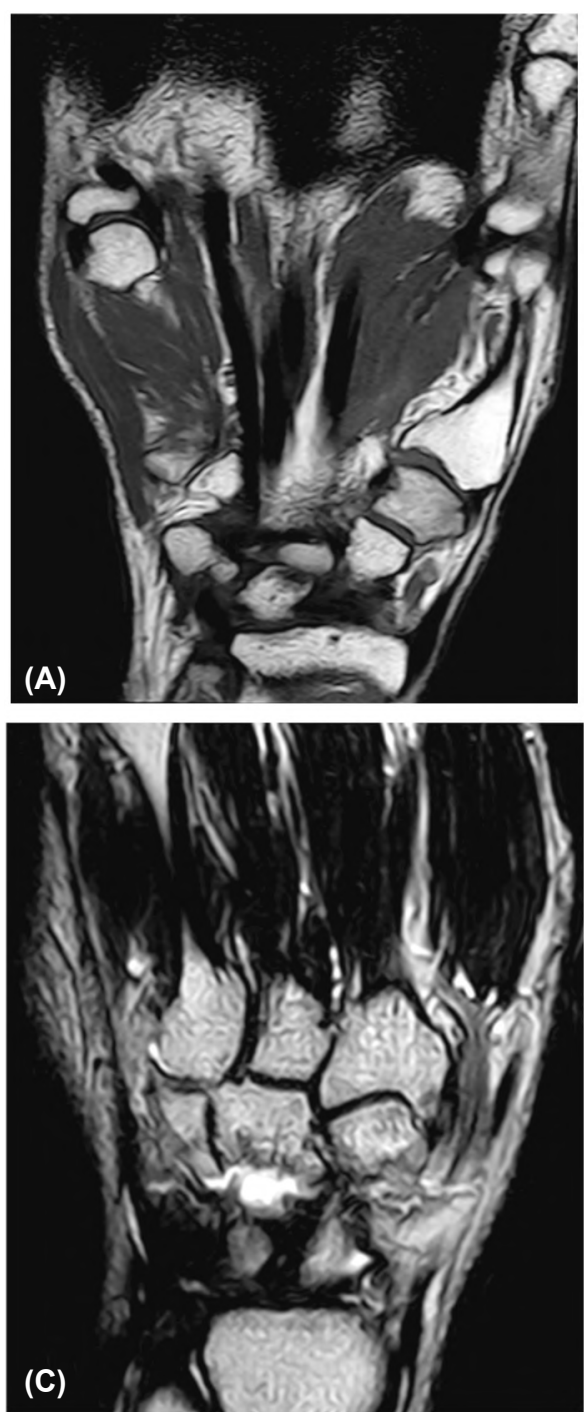
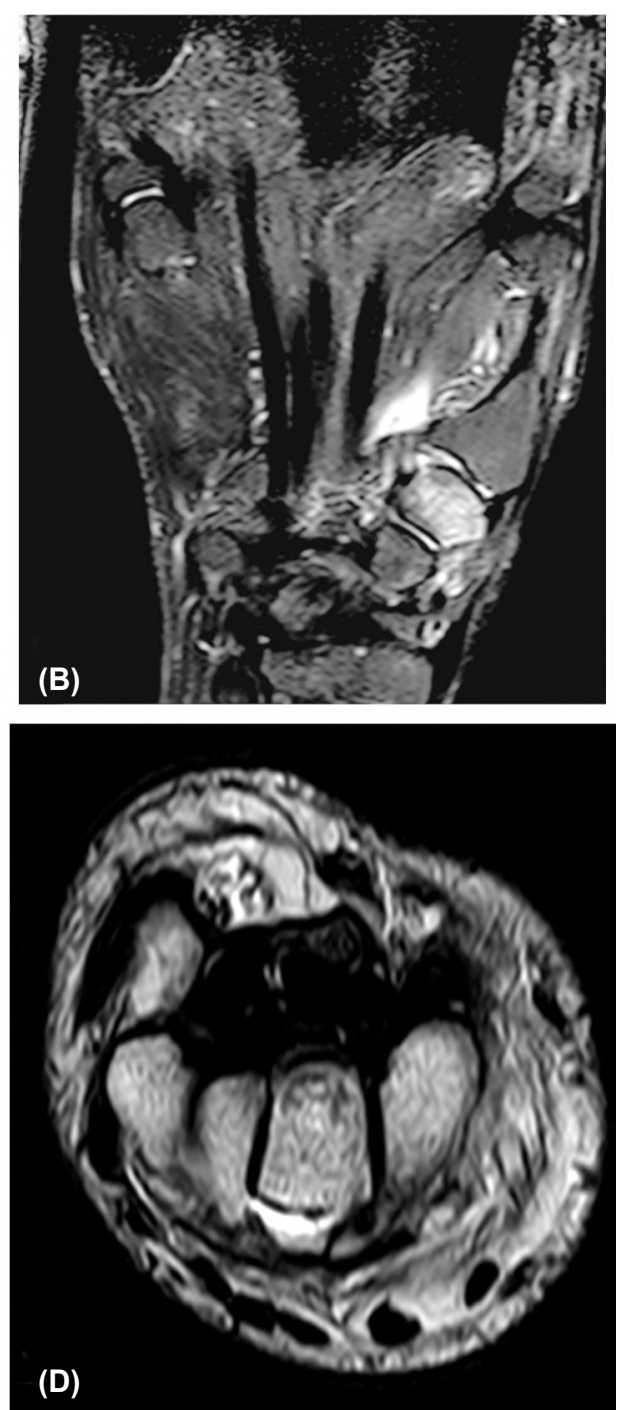

Fig. (2): (A) T1WI showing BME at trapezium with joint effusion and mild synovitis. (B) STIR: the BME appeared clearly with joint effusion \& mild synovitis. (C) Coronal T2WI showing joint effusion at intercarpal joint around capitate and mild synovitis. (D) Axial T2W image showing joint effusion at intercarpal joint around capitate and mild synovitis around the carpal arch.

\section{Discussion}

The diagnosis of RA is largely and collectively based on the interpretation of the signs and symptoms with the use of the 1987 ACR or the 2010 ACR/EULAR classification criteria, laboratory investigation of inflammatory markers, and conventional radiography [7]. Yet, the clinical and laboratory methods are not sufficiently sensitive nor specific in diagnosing the disease. Da Mota et al., found that $30 \%-50 \%$ of patients might be seronegative for RF during the early stage of RA. Similarly, markers such as erythrocyte sedimentation rate and C-reactive protein may be normal in up to $20 \%-25 \%$ of cases [8].

Conventional radiography has the ability of defining bone erosions and joint space narrowing but they are not sensitive enough to detect changes early in the disease process, and it does not provide any information about disease activity or the state of soft tissue component of the joint. $70 \%$ of patients may have normal radiographs at presentation, and erosions may not be revealed by radiography until disease has been established for 12 months or more [9]. This increase the demand for reliable and sensitive methods for early diagnosis and monitoring of the disease.

MRI has increasingly been used in the diagnosis of RA due to its ability to detect the pathological changes in all tissues involved in RA, and many studies showed its greater sensitivity than both clinical examination and X-ray in identifying the inflammatory and destructive change [1]. It depicts active synovitis and bone erosions which are the 
key pathologic features of the disease at presentation which can help establishing an earlier diagnosis for RA. It also allows the detection of tenosynovitis, joint effusions and bone marrow edema (BME) [6].

In our study we imaged patients that had symptoms related to RA without any radiographic abnormalities at time of complain. Some of them had negative rheumatoid factor ( 9 patients, 36\%), 6 of them showed pathological changes in MRI images involving the bones and soft tissues of their wrists and MCP joints. Similarly, some patient had insignificant elevation in ESR and CRP [7 (28\%), 5 (20\%) respectively], though, only 3 of each didn't show any signs in MRI.

Wrist and MCP joints was conducted in this study as they represent the most common and earlier joints affected in RA. Dakkak et al., concluded that the presence of MRI-detected inflammatory features in the foot, didn't have additional value to MRI-detected inflammatory features in the hand in detecting patients who progressed to RA [10]

The earliest abnormality to appear in RA is synovitis. In a prospective study conducted by Sugimoto et al., the use of MRI for detection of synovitis in hands and wrists have shown some improvement in diagnostic accuracy (94\% vs. $83 \%$ ) in early undifferentiated arthritis patients [11].

MRI was able to detect synovial thickening in 20 of 25 patients conducted in this study (80\%). It showed more sensitivity than the classification criteria as it detected synovial thickening in the joints of 2 patients who did not match the classification criteria and assumed not to have RA. This suggest that adding MRI detected synovitis to the scoring system will increases its sensitivity in diagnosis.

The fat-suppressed T2 sequence more clearly described the extent of synovitis, and helped in differentiating it from synovial fluid, as synovitis had a lower signal intensity than joint effusion. After the intravenous administration of gadoliniumbased contrast agent the synovitis enhances rapidly with great intensity. Contrast enhanced imaging assess the activity of synovial inflammation and 12 patients (48\%) showed signs of inflammatory activity at the thickened synovium. The activity of synovitis is a predictor of progressive joint destruction. Also using contrast can differentiate synovitis from joint effusion, which enhance after $6-11 \mathrm{~min}$ as contrast reaches the synovial fluid.
MRI is the only modality that is able to depict BME. It accompanies synovitis in the majority of RA patients, even it could be the only area involved in early RA [6]. Inspite that BME is not diseasespecific, it's clinically meaningful when seen on MRI in both early and late RA, as it associates with future development of bone erosions and poor functional outcomes [3].

17 patients (68\%) were found to have BME in their joints, in 1 case it was the only finding. The different sequences and planes of imaging enabled the assessment of all the small bones of the wrist from different views.

MRI could provide an early diagnosis of RA by reveal erosions, which have been shown to be predictive of later progression in cohorts followed for up to six years [12]. Erosions manifested on MRI as loss of normal low signal intensity of cortical bone on T1-weighted images and loss of normal high signal intensity of the bone marrow cavity. It enhanced after gadolinium administration, and showed high signal intensity on T2-weighted and STIR images. The contrast enhancement of erosions is indicative of the presence of inflamed synovium and is useful in differentiating them from fluid-filled cystic lesions [13]. In STIR images, it was possible to differentiate erosions from the nutrient foramina in the carpal bones or the insertions of interosseous ligament at the volar aspect of the carpal bones that may be shown in some sequences and may be mistaken for small erosions (Fig. C,D,E of case 1).

Rahmani [14] found that the sensitivity of MRI in detection of bone erosion was $98 \%$, and US was $63 \%$, whereas conventional radiography sensitivity in detection of bone erosion was not more than $13 \%$. Therefore, MRI is increasingly being favored in early detection of bone erosions especially in patients with early signs of arthritis. The use of MRI provides more diagnostic facility in earlier diagnosis of RA and differentiating RA from nonRA diseases .

The statistical correlation of synovitis and BME with the 2010 ACR/EULA scoring system was insignificant. But it should be noticed that the false positive value of synovitis and BME represent the presence of inflammatory finding in patients who didn't meet the classification criteria at time of examination and their score was less than 6 , that indicating the higher sensitivity of MRI over the clinical scoring system.

The statistical analysis showed high degree of significance for total positive MRI findings when 
statistically correlated to the 2010 ACR/EULAR classification scoring system used for clinical diagnosis of patients with undifferentiated arthritis, and MRI detected inflammatory signs in 2 patients who did not fulfil the clinical criteria. This add to the high sensitivity of MRI in detecting inflammatory signs and define the extent of the disease. This suggest that adding MRI to the 2010 ACR/EULAR classification scoring, will significantly increase its sensitivity and accuracy in diagnosing RA.

\section{Conclusion:}

The high sensitivity of MRI in detecting inflammatory signs, and the significant correlation with clinical and laboratory findings, indicates the potential importance of MRI study in diagnosing RA early in its course, and the assessment of disease activity that helps in prediction of joint destruction.

\section{References}

1- MOLLER-BISGAARD S., PETERSEN H.K., EJBJERG B.J., BOESEN M., HETLAND M.L., CHRISTENSEN R., MØLLER J., KROGH N.S., PEDERSEN S.K. and ØSTERGAARD M.: Impact of a magnetic resonance imaging-guided treat-to-target strategy on disease activity and progression in patients with rheumatoid arthritis: Study protocol for a randomized controlled trial. Trials, 16: 178, 2015.

2- VENABLES P.J.W. and MAINI R.N.: Clinical features of rheumatoid arthritis. O'Dell J.R., Romain P.R. (eds.): Up-to-date. Wolters Kluwer Health 2016 Accessed at: www.uptodate.com.

3- ISSAR P., NADIGER V. M., HIRAN S. and ISSAR S. K.: Ultrasound and Magnetic Resonance Imaging Correlation of the Wrist and Metacarpophalangeal Joints in Fifty Consecutive Patients of Rheumatoid Arthritis. Indian Journal of Rheumatology, 4: 186-191, 2016.

4- https://www.assh.org/ 2017.

5- XU H., ZHANG Y., ZHANG H., WANG C. and MAO P.: Comparison of the clinical effectiveness of US grading scoring system vs MRI in the diagnosis of early rheumatoid arthritis. Journal of Orthopedic Surgery and Research, 12: 152, 2017.
6- SUDOL-SZOPIN'SKA I., JANS L. and TEH J.: Rheumatoid arthritis: What do MRI and ultrasound show? Journal of Ultrasonography, 17: 5-16, 2017.

7- ALETAHA D., NEOGI T., SILMAN A.J., FUNOVITS J., FELSON D.T., BINGHAM C.O. and BIRNBAUM N.S.: 2010 Rheumatoid Arthritis Classification Criteria: An American College of Rheumatology/European League Against Rheumatism Collaborative Initiative. Arthritis and Rheumatism., 62 (9): 2569-2581, 2010.

8- Da MOTA L.M., CRUZ B.A., BRENOL C.V., PEREIRA I.A., REZENDE-FRONZA L.S., BERTOLO M.B., et al.: Guidelines for the drug treatment of rheumatoid arthritis. Revista Brasileira de Reumatologia, 53 (2): 158-183, 2013.

9- McQUEEN F.M., CHAPMAN P., POLLOCK T., D'SOUZA D., LEE A.C., DALBETH N., STAMP L., LINDSAY K. and DOYLE A.: Changes in clinical disease activity are weakly linked to changes in MRI inflammation on treat-to-target escalation of therapy in rheumatoid arthritis. Arthritis Research \& Therapy, 19: 241-245, 2017.

10- DAKKAK Y.J., BOETERS D.M., BOER A.C., REIJNIERSE M. and VAN DER HELM-VAN MIL A.H.M. What is the additional value of MRI of the foot to the hand in undifferentiated arthritis to predict rheumatoid arthritis development? Arthritis Research \& Therapy, Volume, 21 (56), 2019.

11- SUGIMOTO H., TAKEDA A. and HYODOH K.: Earlystage rheumatoid arthritis: Prospective study of the effectiveness of MR imaging for diagnosis. Radiology, 216 (2): 569-75, 2000.

12- Da SILVA R.M., De SOUSA D.S., Da SILVA F.S., PINTO Y.M.R., COSTA D.L., PINHEIRO M. and LIMA V.H.A.: Rheumatoid Arthritis in Image of Magnetic Resonance: A Proposal for Review of Literature. International Journal of Tropical Disease \& Health, 31 (4): 1-11, 2018.

13- TAVARES-JÚNIOR W.C., ROLIM R. and KAKEHASI A.M.: Magnetic resonance imaging in rheumatoid arthritis. Revista brasileira de reumatologia, 51 (6): 635-641, 2011.

14- RAHMANI M., CHEGINI H., NAJAFIZADEH S. R., AZIMI M., HABIBOLLAHI P. and SHAKIBA M.: Detection of bone erosion in early rheumatoid arthritis: Ultrasonography and conventional radiography versus non-contrast magnetic resonance imaging. Clinical Rheumatology, 29 (8): 883-91, 2010. 


\section{دور التصوير بالرنين المغناطيسى فى تشخيص ومتابعة مرض إلتهاب المفاصل التهل الروماتيزمى}

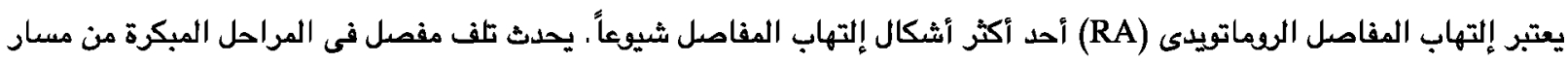

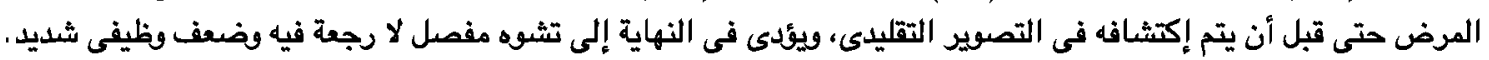

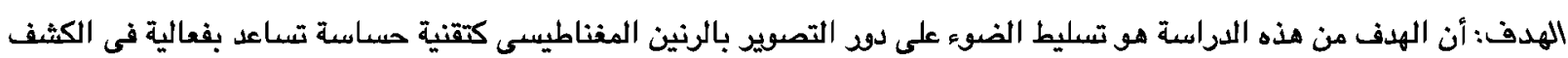

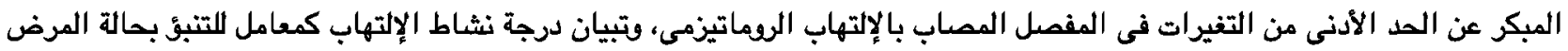

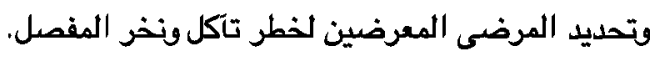

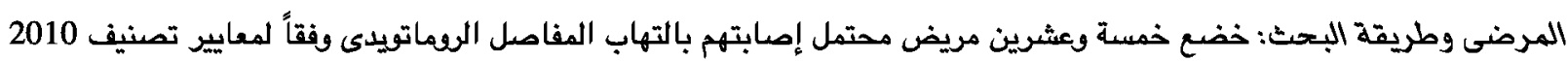

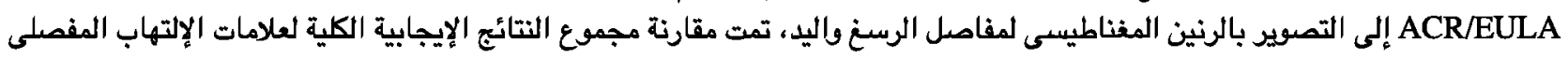

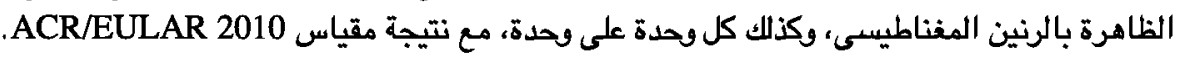
النتائج والإستتاج: وجدنا ارتباطاً كبيراً واتفاقاً بين النتائج الإيجابية الكلية للرنين المفناطيسى ومعائير

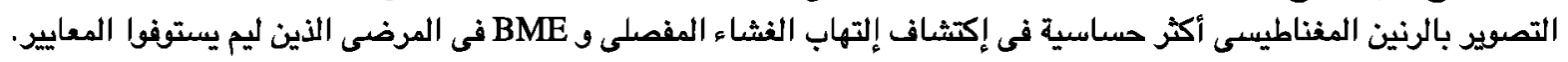

